University of Wollongong

Research Online

Australian Institute for Innovative Materials -

Papers

Australian Institute for Innovative Materials

$1-1-2016$

Magnetocaloric effect in HoMn2Si2 compound with multiple magnetic phase transitions

J C. Debnath

Deakin University, jcd341@uow.edu.au

Jian Li Wang

University of Wollongong, jianli@uow.edu.au

Follow this and additional works at: https://ro.uow.edu.au/aiimpapers

Part of the Engineering Commons, and the Physical Sciences and Mathematics Commons

Research Online is the open access institutional repository for the University of Wollongong. For further information contact the UOW Library: research-pubs@uow.edu.au 


\title{
Magnetocaloric effect in HoMn2Si2 compound with multiple magnetic phase transitions
}

\author{
Abstract \\ The structural, magnetic and magnetocaloric properties of the polycrystalline compound HoMn2Si2 have \\ been studied. The temperature variation of magnetization and heat capacity show that below room \\ temperature the compound under goes multiple magnetic transitions, at about 80,15 and $3.5 \mathrm{~K}$ \\ respectively. Moreover, the presence of the interesting thermomagnetic irreversibility in $\mathrm{HoMn} 2 \mathrm{Si} 2$ is \\ detected in the magnetization versus temperature plot, which can be ascribed to the narrow domain wall \\ pinning effect. A broad and asymmetric peak is observed for the MCE response which might suggest the \\ underlying first-order nature of the transition and/or the spin fluctuations of the Mn subsystem. The \\ density of states $\mathrm{N}(\mathrm{EF})$ at the Fermi level and the Debye temperature have been determined and analyzed. \\ Large magnetocaloric effect $(\Delta S M=-9 \mathrm{~J} / \mathrm{kg}-\mathrm{K}$ for a field change of $5 \mathrm{~T}$ around $9 \mathrm{~K})$ suggest that this \\ material is suitable for the low temperature magnetic refrigeration.

\section{Disciplines} \\ Engineering | Physical Sciences and Mathematics

\section{Publication Details} \\ Debnath, J. C. \& Wang, J. Li. (2016). Magnetocaloric effect in HoMn2Si2 compound with multiple \\ magnetic phase transitions. Intermetallics, 78 50-54.
}


Magnetocaloric effect in $\mathrm{HoMn}_{2} \mathrm{Si}_{2}$ compound with multiple magnetic phase transitions

J. C. Debnath ${ }^{* 1}$ and Jianli Wang ${ }^{2}$

${ }^{1}$ Institute for Frontier Materials, Deakin University, Geelong, VIC 3216, Australia

${ }^{2}$ Institute for Superconducting \& Electronic Materials, University of Wollongong, New South Wales, 2522, Australia.

Corresponding author: jcd341@uowmail.edu.au; Jyotish.debnath@ deakin.edu.au 


\begin{abstract}
:
The structural, magnetic and magnetocaloric properties of the polycrystalline compound $\mathrm{HoMn}_{2} \mathrm{Si}_{2}$ have been studied. The temperature variation of magnetization and heat capacity show that below room temperature the compound under goes multiple magnetic transitions, at about 80, 15 and $3.5 \mathrm{~K}$ respectively. Moreover, the presence of the interesting thermomagnetic irreversibility in $\mathrm{HoMn}_{2} \mathrm{Si}_{2}$ is detected in the magnetization versus temperature plot, which can be ascribed to the narrow domain wall pinning effect. A broad and asymmetric peak is observed for the MCE response which might suggest the underlying first-order nature of the transition and/or the spin fluctuations of the $\mathrm{Mn}$ subsystem. The density of states $N\left(E_{F}\right)$ at the Fermi level and the Debye temperature have been determined and analyzed. Large magnetocaloric effect $\left(\Delta S_{M}=-9 \mathrm{~J} / \mathrm{kg}-\mathrm{K}\right.$ for a field change of $5 \mathrm{~T}$ around $9 \mathrm{~K}$ ) suggest that this material is suitable for the low temperature magnetic refrigeration.
\end{abstract}


Keywords: Intermetallics; $\mathrm{HoMn}_{2} \mathrm{Si}_{2}$ compound; Magnetocaloric effect; Magnetic phase transition;

\section{Introduction}

In recent years, the magnetic refrigeration, a green cooling technology based on magnetocaloric effect (MCE), an intrinsic phenomenon of a magnetic material has attracted much attention due to its higher energy efficiency and eco-friendliness than the commonly used conventional gas compression refrigeration which uses a magnetic field to change the magnetic entropy of a material [1-6]. The MCE is an intrinsic phenomenon of magnetic materials, which manifests as an isothermal magnetic entropy change $\left(\Delta S_{M}\right)$ or an adiabatic temperature change $\left(\Delta T_{a d}\right)$ when the magnetic material is exposed to a varying magnetic field. Large values of $\Delta S_{M}$ and $\Delta T_{a d}$ under a varying magnetic field are considered to be the most important indicator of magnetic cooling materials for the practical application, and therefore it is important to find new materials with large MCE. So to search for magnetic materials with large values $\Delta S_{M}$ and $\Delta T_{a d}$ is the main mission in this field of research.

Typically, the large/giant MCE is observed in the material that undergoes first order magnetic phase transition. Large number of magnetic materials with large entropy change accompanied by a first order phase transition have been reported [1-3, 7]. Apart from MCE, the first order magnetic transition (FOMT) gives rise to various other functional properties of technological importance, such as magnetostrictive, magnetoresistance, shape memory effects etc. [8]. However, the first-order phase transition is usually accompanied by considerable thermal and/or magnetic hysteresis along with irreversibility, which is disadvantage for a magnetic refrigeration cycle [7, 9-11]. But some of them, especially rare-earth-based intermetallic compounds, have reversible large MCE along with a small hysteresis loss [12-15]. Therefore 
it is desirable to search for rare-earth-based intermetallic materials that not only exhibit large reversible MCE but also have none or negligible thermal and field hysteresis loss.

The $R T_{2} X_{2}$ compounds, where $R$ is rare earth intermetallic compounds (Gd, Tb, Dy, Ho etc.), $T$ is transition metal, and $X$ is $\mathrm{Si} / \mathrm{Ge}$, is an important class of materials, which exhibits a wide variety of interesting physical properties [16-19]. Depending on the constituent element or composition, various properties like superconductivity, magnetic ordering, heavy-fermion properties, valence fluctuation, large MCE, etc. are observed [16-19]. As Mn carries magnetic moment, (unlike many other $\mathrm{T}$ elements such as $\mathrm{Co}, \mathrm{Ni}$ and $\mathrm{Fe}$ ) and its magnetic states strongly depends on intraplanar Mn-Mn distances, the compounds with $\mathrm{T}=\mathrm{Mn}$ attract particular interests $[16,20]$. It is also known that, only in the case where $\mathrm{T}=\mathrm{Mn}$ do they display long-range magnetic order, which, incidentally can exist even above $300 \mathrm{~K}$. However, the rare earth lattice orders magnetically at lower temperatures. The $\mathrm{RMn}_{2} \mathrm{X}_{2}$ compounds crystallize in the $\mathrm{ThCr}_{2} \mathrm{Si}_{2}$-type body centered tetragonal structure (with space group $I 4 / \mathrm{mmm})[21,22]$ with repeated layers of atoms stacked in the sequence $R-X-M n-X-R$ [20, 23] along the c-axis (similar to $R_{5}(\mathrm{Si}, \mathrm{Ge})_{4}$, a family of well-known large MCE materials) [24]. These characteristics of $R T_{2} X_{2}$ compounds offer scopes for selection of the magnetic stateand therefore the MCE - by controlling the interlayer and intralayer distances between magnetic atoms.

Recently, some $R T_{2} X_{2}$ compounds have been observed to possess not only large MCE but also a small hysteresis loss around their ordering temperature [18, 19]. The magnetic structure of $\mathrm{HoMn}_{2} \mathrm{Si}_{2}$ material has been studied using neutron diffraction dates from the 1990s [25]. This compound exhibits the crystal structure of $\mathrm{ThCr}_{2} \mathrm{Si}_{2}$ type with the $\mathrm{Z}$ parameter of $\mathrm{Si}$ in $4(\mathrm{e})$ site (space group $14 / \mathrm{mmm}$ ). It exhibits antiferromagnetic ordering in the form of a flat spiral of Ho moments. Mn magnetic moments located in planes normal to 
the c-axis are coupled ferromagnetically, but moments in adjacent planes can be coupled either ferromagnetically or antiferromagnetically. They are aligned along the $\mathrm{c}$ axis.

In order to improve the application of magnetocaloric cooling, efforts have been paid to search for magnetic refrigerants in view of domestic or industrial applications near room temperature [1-7]. On the other hand, the research on systems exhibiting large MCEs at low temperature is also important due to their prospective application in the refrigeration for gas liquefaction of hydrogen and helium [26-28]. Magnetic refrigeration at liquid helium temperatures is indeed of interest especially in space, medical and other cryogenic applications [29]. Significant capital cost reduction can potentially be achieved with a magnetic hydrogen liquefier compared to conventional liquefiers. Magnetic refrigeration may allow hydrogen to be competitive as an alternative fuel source [30].

In this paper, for achieving a complete understanding of $\mathrm{HoMn}_{2} \mathrm{Si}_{2}$ material, a detailed investigation of the magnetic structure and magnetic phase transitions was presented. A large reversible MCE was observed, accompanied by a first order magnetic phase transition which is applicable for low temperature magnetic refrigeration. Characteristics of the magnetic transition, the origin of large MCE as well as its potential application in $\mathrm{HoMn}_{2} \mathrm{Si}_{2}$ were discussed.

\section{Experimental details}

Polycrystalline $\mathrm{HoMn}_{2} \mathrm{Si}_{2}$ sample was synthesised by an arc melting method under an argon atmosphere. The stoichiometric amounts of high purity Ho (99.9\%), Mn (99.9 \%) and Si $(99.99 \%)$ were melted four times for homogeneity on a water-cooled copper hearth. Around 3\% excess Mn was added to compensate the Mn evaporation during the melting. The as-cast samples were sealed in an evacuated quartz tube and annealed for 1 week at $800^{\circ} \mathrm{C}$ followed by furnace cooling. The sample was proved to be single phase with the body 
centered tetragonal $\mathrm{ThCr}_{2} \mathrm{Si}_{2}$ type structure by x-ray powder diffraction experimental (XRD) which employs $\mathrm{Cu} \mathrm{K}-\alpha$ radiation. The Rietveld refinements were carried out using the Fullprof program. Magnetic measurements, including temperature dependent magnetization curves and magnetization-field loops were performed in the temperature range of 2-300 K and field range of 0 - $7 \mathrm{~T}$ using a SQUID Magnetic Property Measurement System (MPMS) from Quantum Design Inc., San Diego. Heat capacity measurements were conducted on a Quantum Design 14 T physical properties measurement system.

\section{Results and discussion}

A schematic of the crystal structure of $\mathrm{HoMn}_{2} \mathrm{Si}_{2}$ is provided in Fig. 1 along with Rietveld refinement of $x$ ray data. The sample is confirmed to be single phase with the body centered tetragonal $\mathrm{ThCr}_{2} \mathrm{Si}_{2}$ type structure (space group I4/mmm). The calculated lattice parameters at room temperature $\left[\mathrm{a}(\AA)=3.922\right.$, $\mathrm{c}(\AA)=10.444$ and $\left.\mathrm{V}\left(\AA^{3}\right)=16.044\right]$ derived from the refinement are in good agreement with the reported values [25, 31]. There is small amount of second phase present in the sample. According to ref. 32, the peak around 32 degree of 2 theta may be ascribed to the presence of $\mathrm{Ho}_{4} \mathrm{Si}_{3} \mathrm{O}_{12}$ phase [32].

The temperature dependence of the magnetization $(M)$ in the temperature $(T)$ range of 5$150 \mathrm{~K}$ in an applied field of 500 Oe under zero field cooling (ZFC) and field cooling (FC) process, is shown in Fig. 2. Multiple phase transitions have been detected. Similar behaviors had been reported in the compound $\mathrm{TbMn}_{2} \mathrm{Si}_{2}$ [33]. A major magnetic phase transition occurs around $80 \mathrm{~K}$ where $\mathrm{HoMn}_{2} \mathrm{Si}_{2}$ seems to enter the magnetically ordered state from antiferromagnetic state (based on ref. 20 where it was reported that the interlayer antiferromagnetic state forms around $\mathrm{T}_{\mathrm{N}}=453 \mathrm{~K}$ ). Further, two more minor anomalies are present around at $\sim 15 \mathrm{~K}$ and at $3.5 \mathrm{~K}$ in the ZFC magnetization data. According to ref. 25, the transition at $15 \mathrm{~K}$ can be ascribed to the formation of a flat spiral of Ho moment. The 
reflection of the transition at temperature $3.5 \mathrm{~K}$ may be attributed to the ordering of $\mathrm{Ho}^{3+}$ moments but a detailed study is warranted. In order to test if the coexistence of multiple phases occurs, we measured thermal hysteresis in magnetization as shown in the top inset of Fig. 2.

There exists a definite thermal hysteresis below $20 \mathrm{~K}$ which might suggest the presence of multiple magnetic phases or structures at low temperatures. This is also a generic feature associated with the first order magnetic transition (FOMT). Usually, smaller thermal hysteresis is expected in the case of a first order magneto-elastic transition [34], On the other hand, a huge thermal hysteresis is observed in the case of magnetic transition connected with a martensitic-like structural transition. For the present compound the observed thermal hysteresis is very small. Therefore, the thermal hysteresis observed in $\mathrm{HoMn}_{2} \mathrm{Si}_{2}$ compound suggests that the magnetic transitions are not connected with structural change, but simply an iso-structural volume change associated with the magnetic transitions. This statement is consistent with the conclusion derived based on the available neutron results in [25] where no structural changes have been detected below room temperature for $\mathrm{HoMn}_{2} \mathrm{Si}_{2}$.

The inverse magnetic susceptibility, $1 / \chi(T)$, was fitted with a modified Curie-Weiss law in the paramagnetic region $180-300 \mathrm{~K}$ (not shown) following the expression $1 / \chi(T)=\chi_{0}$ $+C /\left(T-\theta_{p}\right)$ where $\chi_{0}$ is the temperature independent term and $\theta_{p}$ is the Curie-Weiss temperature. The evaluated value of effective magnetic moment is $\mu_{\text {eff }}=12.27 \mu_{\mathrm{B}}$ and $\theta_{p}=-64 \mathrm{~K}$ suggestive of antiferromagnetic correlations. The observed $\mu_{\text {eff }}$ is larger than the moment of free $\mathrm{Ho}^{3+}$ which is $10.6 \mu_{\mathrm{B}}$, suggests that the magnetic interaction in this compound is not only due to the rare earth ion but also there are contributions from the moment on $\mathrm{Mn}$ and from the effects of spin polarization of conduction electrons which is termed as magnetic polaronic effect [35]. The magnetic polarons mainly arises in compounds 
with strong $s-f$ exchange. This value is in good agreement with the expected value;

$$
\mu_{\text {eff }}=\sqrt{ }\left[\left\{\mu_{\text {eff }}\left(H o^{3+}\right)\right\}^{2}+\left\{\mu_{e f f}\left(M n^{3+}\right)\right\}^{2}+\left\{\mu_{e f f}\left(M n^{4+}\right)\right\}^{2}\right]=12.35 \mu_{\mathrm{B}} .
$$

For the $\mathrm{RMn}_{2} \mathrm{X}_{2}$ systems, it is well accepted that the magnetic ordering of $\mathrm{Mn}$ moments persists to the temperature above $300 \mathrm{~K}$ while magnetic ordering on rare earth occurs at low

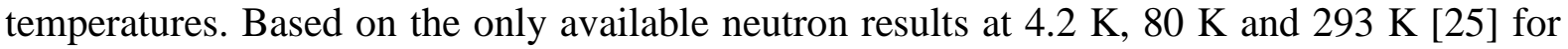
$\mathrm{HoMn}_{2} \mathrm{Si}_{2}$, it can be assumed that the $\mathrm{Mn}$ sublattices moment already forms a antiferromagnetic structure along c-axis at $293 \mathrm{~K}$ where the magnetic peaks (111) and (113) are present. While the Ho sublattice moment forms an incommensurate magnetic structure only below $80 \mathrm{~K}$ with a doubled flat spiral due to the fact that at $80 \mathrm{~K}$ no satellite peaks are present at $80 \mathrm{~K}$ neutron pattern [25]. Based on the fact there are no detailed neutron results in hand, we can only assume that the transition at $15 \mathrm{~K}$ corresponds to the formation of incommensurate magnetic structure (see the inset of Figure 1 in ref. [25] where the intensity of peak $(001 \pm)$ drops sharply). However, due to the strong interaction between the moment from rare earth sublattice and $\mathrm{Mn}$ sublattice as detected in $\mathrm{TbMn}_{2} \mathrm{Si}_{2}[33,36]$ and $\mathrm{DyMn}_{2} \mathrm{Si}_{2}$ [37], it should be expected that both Ho-sublattice and Mn sublattice will contribute to all the three magnetic transitions. In order to get a fully understanding these magnetic phase transitions, a detailed neutron diffraction experiment at variable temperatures is needed. Apart from the multiple magnetic transitions, another feature worth noting from Fig. 2 is the occurrence of thermomagnetic irreversibility between the FC and ZFC magnetization data. It is clearly seen that the ZFC and FC curves above $15 \mathrm{~K}$ are completely reversible, whereas a distinct discrepancy between ZFC and FC curves appears below this temperature. This thermomagnetic irreversibility can be observed in many cases such as spin-glass systems, materials with competing magnetic interactions, and ferromagnetic materials with high anisotropy [38-40]. 
In order to explore thermomagnetic irreversibility, the magnetic hysteresis loop of $\mathrm{HoMn}_{2} \mathrm{Si}_{2}$ at $2 \mathrm{~K}$ is measured and presented in the bottom inset of Fig. 2. A hysteresis with coercive field of $0.05 \mathrm{~T}$ is observed, confirming the anisotropy of $\mathrm{HoMn}_{2} \mathrm{Si}_{2}$ at low temperature. For the materials with high anisotropy and low ordering temperature, the narrow domain walls could exist, thus resulting in a large pinning effect [40]. In ZFC mode, the domain walls are pinned, and the thermal energy is not strong enough to overcome the energy barriers. This leads to the lower magnetization. On the other hand, in FC mode, the magnetic domains are already oriented along the applied magnetic field direction. As the domain walls move more easily when the temperature increases and therefore the magnetization at low temperature is higher than that in ZFC mode. Considering the magnetic anisotropy, it is anticipated that the thermomagnetic irreversibility between $\mathrm{ZFC}$ and $\mathrm{FC}$ curves seen in $\mathrm{HoMn}_{2} \mathrm{Si}_{2}$ is likely attributed to the narrow domain wall pinning effect.

A set of magnetic isotherms were measured for $\mathrm{HoMn}_{2} \mathrm{Si}_{2}$ with the temperature range 2 to 40 $\mathrm{K}$ up to $5 \mathrm{~T}$ applied field as shown in Fig. 3 (a). A negligible hysteresis is observed for all the temperature range which may confirm a reversible MCE for that compound. It is apparent that the magnetization tends to saturate for sufficiently high magnetic fields. However, the attained value at $2 \mathrm{~K}(87 \mathrm{emu} / \mathrm{g}$ for $5 \mathrm{~T})$ reaches only $31 \%$ of that expected for a complete alignment of the magnetic moments of $\mathrm{Ho}^{3+}\left(10 \mu_{\mathrm{B}}\right), \mathrm{Mn}^{3+}\left(4 \mu_{\mathrm{B}}\right)$, and $\mathrm{Mn}^{4+}\left(3.8 \mu_{\mathrm{B}}\right)$, which is equivalent to a magnetization of $280 \mathrm{emu} / \mathrm{g}$.

As we know that the magnitude of MCE has a strong correlation with the nature of the corresponding magnetic phase transition. So it is important to understand the order of magnetic transition in $\mathrm{HoMn}_{2} \mathrm{Si}_{2}$. The measured Arrott plot for the compound $\mathrm{HoMn}_{2} \mathrm{Si}_{2}$ is shown in Fig. 3(b). The S-shaped nature of the Arrott plot denotes the negative sign of the 
coefficient $c_{2}(\mathrm{~T})$ in the Landau expansion of the magnetic free energy, confirming the characteristic of a first order magnetic transition according to Banerjee criterion [41].

Temperature dependence of the heat capacity $C_{p}$ for $\mathrm{HoMn}_{2} \mathrm{Si}_{2}$ in the temperature range of 0.5 to $150 \mathrm{~K}$ is shown in the Fig. 4. Two major anomalies are observed at $\sim 18 \mathrm{~K}$ and at $4 \mathrm{~K}$ respectively (see Fig. 4(a)) which are consistence with the magnetization data. We observed the sign of anomaly around $80 \mathrm{~K}$ in the heat capacity even it is not less obvious compared with the two at lower temperatures (at $\sim 18 \mathrm{~K}$ and at $4 \mathrm{~K}$ ). In general, the heat capacity of materials with a first order magnetic phase transition exhibits a fairly sharp peak near the transition temperature while a $\lambda$-type anomaly is present around transition temperature in materials with a magnetic phase transition of second order. The size and shape of heat capacity anomaly may also depend on the sample purity and homogeneity. The almost absence of anomaly around $80 \mathrm{~K}$ here may also be ascribed to the small sensitivity of the PPMS device and type of this particular transition as described in ref [42] where similar behaviours occur for $\mathrm{La}_{0.75} \mathrm{Y}_{0.25} \mathrm{Mn}_{2} \mathrm{Si}_{2}$ that a clear anomaly is detected for $\mathrm{T}_{\mathrm{C}}$ but no obvious anomaly is present at $\mathrm{T}_{\mathrm{AF}}$.

Fig. 4 (b) draws the very low temperature heat capacity for the temperature range $0.5-3 \mathrm{~K}$ in order to confirm the presence of any more anomalies. No anomaly is observed at that temperature range. As we know that the total heat capacity is the total contributions from the electrons, $C_{e l}(T)$, phonons, $C_{p h}(T)$, and magnons, $C_{m}(T)$. At lower temperature region the main contribution comes from the electrons and phonons. In order to estimate the electrons contribution to specific heat we use an equation described as:

$$
C_{P}=\gamma T+\beta T^{3}
$$

where the first tem represents the contribution of electrons and second term comes from the contribution of phonons. $\gamma$ is given by:

$$
\gamma=\pi^{2} k_{B}^{2} N\left(E_{F}\right) / 3
$$


Here, $N\left(E_{F}\right)$ is the electronic density of states of at the Fermi level and $k_{B}$ is the Boltzman constant.

To estimate the value for $\gamma$, a plot of $C_{p} / T$ versus $T^{2}$ at lower temperatures $(\leq 12 \mathrm{~K})$ is derived and shown in Fig. 4(c). The estimated values for $\gamma$ is equal to $99 \mathrm{~mJ} / \mathrm{mol} \mathrm{K} \mathrm{K}^{2}$ and the Debye temperature, $\Theta_{D}=157 \mathrm{~K}$ respectively. The estimated $\gamma$ leads to the estimate of density of states at the Fermi level as $N\left(E_{F}\right)=8.4$ states/eV (using equation 2). Compared with the $\gamma$ values and $\mathrm{N}\left(E_{F}\right)$ derived for $\mathrm{LaMn}_{2} \mathrm{Si}_{2}\left(\gamma=33 \mathrm{~mJ} / \mathrm{mol} K^{2}\right.$ and $\mathrm{N}\left(E_{F}\right)=2.82$ states/eV atom $)$ [42], $\mathrm{YMn}_{2} \mathrm{Si}_{2}\left(\gamma=18 \mathrm{~mJ} / \mathrm{mol} K^{2}\right.$ and $\mathrm{N}\left(E_{F}\right)=1.52$ states/eV atom) [42] and $\mathrm{TbMn}_{2} \mathrm{Si}_{2}(\gamma=28$ $m J / m o l K^{2}$ and $\mathrm{N}\left(E_{F}\right)=2.38$ states/eV atom) [36] compounds, this remarkable large $\gamma$ value may be due to an enhanced electronic density of states at the Fermi level.

It is observed that there is an increase of $C_{p} / T$ at lower temperature inset in Fig. 4. This increase at lower temperature can be ascribed to the contribution from the nuclear specific heat $\mathrm{C}_{\mathrm{N}}$ that arises due to the splitting of the nuclear hyperfine levels as described for $\operatorname{PrAl}_{2}$ in [43] where it was found that heat capacity becomes enhanced at $\mathrm{T}<4 \mathrm{~K}$. This increase of $C_{p} / T$ here for $\mathrm{HoMn}_{2} \mathrm{Si}_{2}$ is consistent with the discovery of large nuclear specific heat $\mathrm{C}_{\mathrm{N}}$ in some of the lanthanides. It is accepted that the interactions of the nuclear magnetic moments with the strong magnetic field produced by the $4 \mathrm{f}$ electrons at the sites of the nuclei contributes to the high nuclear specific heat $\left(\mathrm{C}_{\mathrm{N}}\right)$ at low temperature.

The magnetic entropy $-\Delta S_{M}$ for the present compound $\mathrm{HoMn}_{2} \mathrm{Si}_{2}$ was calculated from the magnetization isotherm $M(H, T)$ curves in Fig. 3 (a) using an integral version of Maxwell's thermodynamic relation

$$
\Delta S_{M}(\mathrm{~T}, \mathrm{H})=\int_{0}^{H}\left(\frac{\delta M(T, H)}{\delta T}\right)_{H} d H
$$


The temperature dependence of $-\Delta S_{M}$ for $\mathrm{HoMn}_{2} \mathrm{Si}_{2}$ calculated from $M(H, T)$ data for different magnetic field variations up to $5 \mathrm{~T}$ is shown in Fig. 5. The maximum values of magnetic entropy change reach about 4.8 and $9 \mathrm{~J} / \mathrm{kg} \mathrm{K}$ for the field change of 3 and $5 \mathrm{~T}$ respectively. These obtained values are comparable to larger than those of some potential low temperature magnetic refrigerants such as $\mathrm{Ho}\left(\mathrm{Ni}_{1-\mathrm{x}} \mathrm{Fe}_{\mathrm{x}}\right)_{2}, \mathrm{GdCuSi}$ and $\mathrm{PrCo}_{2} \mathrm{~B}_{2}$ [44-46]. This large magnetocaloric effect is suitable for low temperature magnetic refrigeration. It is clear from Fig. 5 that the $\Delta S_{M}$ peak broadens asymmetrically towards high temperature on increasing the applied magnetic field. Such a behaviour is observed in systems with first-order phase transitions [47], spin-flop transition [48] and spin fluctuations [49].

\section{Conclusions}

To summarize, three magnetic phase transitions have been detected in $\mathrm{HoMn}_{2} \mathrm{Si}_{2}$ below room temperature. An obvious magneto-history effect below $15 \mathrm{~K}$ is present which can be attributed to narrow domain wall pinning effect. First order nature of the transitions is revealed by Arrott plots. Large magnetic entropy change $\Delta S_{M}=-9 \mathrm{~J} / \mathrm{kg}-\mathrm{K}$ is observed at $9 \mathrm{~K}$ with the application of $5 \mathrm{~T}$ magnetic field which is applicable for the low temperature magnetic refrigeration. The low temperature heat capacity has been analysed in details suggesting the presence of large nuclear specific heat contribution.

\section{Acknowledgement}

J. C. Debnath acknowledges the Alfred Deakin Postdoctoral Research Fellowship at Deakin University, Australia.

\section{References}

[1] Dan'kov S Yu, Tishin AM, Pecharsky VK, Gschneidner Jr KA. Phys. Rev. B $1998 ; 57: 3478$ 
[2] Tegus O, Bruck E, Buschow KHJ, de Boer F R. Nature 2002;415:150.

[3] Debnath JC, Zeng R, Kim JH, Dou SX. J. Magn. Magn. Mater. 2011;323:138.

[4] Gutfleisch O, Willard MA, Bruck E, Chen CH, Sankar SG, Liu JP. Adv. Mater. 2011;23:821.

[5] Liu J, Gottschall T, Skokov KP, Moore JD, Gutfleisch O. Nat. Mater. 2012;11:620.

[6] Md Din MF, Wang JL, Zeng R, Shamba P, Debnath JC, Dou SX. Intermettalics 2013;36:1.

[7] Zou JD, Shen BG, Gao B, Shen J, Sun JR. Adv. Mater. 2009;21:693.

[8] Roy SB. J. Phys.: Condens. Matter 2013;25:183201.

[9] Phan MH, Woods GT, Chaturvedi A, Stefanoski S, Nolas GS, Srikanth H. Appl. Phys. Lett. 2008;93:52505.

[10] Sun NK, Cui WB, Li D, Geng DY, Yang F, Zhang ZD. Appl. Phys. Lett. 2008;92:072504.

[11] Moore JD, Morrison K, Sandeman KG, Katter M, Cohen LF. Appl. Phys. Lett. 2009;95:252504.

[12] Shen J, Gao B, Zhang HW, Hu FX, Li YX, Sun JR, Shen BG. Appl. Phys. Lett. 2007;91:142504.

[13] Shamba P, Debnath JC, Zeng R, Wang JL, Campbell SJ, Kennedy SJ, Dou SX. J. Appl. Phys. 2013;113:17A941.

[14] Li LW, Niehaus O, Kersting M, Pöttgen R. Appl. Phys. Lett. 2014;104:092416.

[15] Chaturvedi A, Stefanoski S, Phan MH, Nolas GS, Srikanth H. Appl. Phys. Lett. 2011;99:162513.

[16] Kumar P, Suresh KG, Nigam AK, Magnus A, Coelho AA, Gama S. Phys. Rev. B $2008 ; 77: 224427$

[17] Li L, Nishimura K, Usui G, Huo D, Qian Z. Intermetallics 2012;23:101. 
[18] Wang JL, Campbell SJ, Cadogan JM, Studer A, Zeng R, Dou SX. Appl. Phys. Lett. 2011;98:232509.

[19] Samanta T, Das I, Banerjee S. Appl. Phys. Lett. 2007;91:152506.

[20] Szytula A, Szott I. Solid State Commun. 1981;40:199.

[21] Samanta T, Das I, Banerjee S. Appl. Phys. Lett. 2007;91:152506.

[22] Wang JL, Caron L, Campbell SJ, Kennedy SJ, Hofmann M, Cheng ZX, Md. Din MF, Studer AJ, Brück E, Dou SX. Phys. Rev. Lett. 2013;110:217211.

[23] Rossi D, Marazza R, Mazzone D, Ferro R. J. Less-Common Met. 1978;59:79.

[24] Pecharsky VK, Gschneidner KA Jr. Phys. Rev. Lett. 1997;78:4494.

[25] Leciejewicz J, Szytula A, Baźela W, Siek S. J. Magn. Magn. Mater. 1990;89:29.

[26] Gschneidner KA Jr, Pecharsky VK, Tsokol AO. Rep. Progr. Phys. 2005;68:1479.

[27] Zhang H, Shen BG, Xu ZY, Shen J, Hu FX, Sun JR, Long Y. Appl. Phys. Lett. 2013;102:092401.

[28] Matsumoto K, Kondo T, Yoshioka S, Kamiya K, Numazawa T. In J. Phys.: Conf. Ser., 2009;150:012028.

[29] Zhang L, Sherif SA, DeGregoria AJ, Zimm CB, Veziroglu TN. Cryogenics 2000;40:269.

[30] Scheihing PE. Industrial magnetic refrigeration for hydrogen liquefaction. In: Proceedings of the XVIII ${ }^{\text {th }}$ International Congress of Refrigeration, Montreal, Que., Canada; 10 - 17 August 1991.

[31] Narasimhan KSVL, Rao VUS, Wallace WE, Pop I. AIP Conference Proceedings 1976;29:594.

[32] Kudoua K, Okadab S, Moric T, Iizumid K, Shishidoe T, Tanaka T, Kanari H, Rogl P. J. Alloys Comp. 2003;358:182.

[33] Maji B, Roy MK, Suresh KG, Banerjee S. J. Appl. Phys. 2014;116:213913. 
[34] Skokov KP, Khovaylo VV, Müller KH, Moore JD, Liu J, Gutfleisch O. J. Appl. Phys. 2012;111:07A910.

[35] Ehlers G, Maletta H. Z. Phys. B 1996;99:145.

[36] Li GX, Wang JL, Cheng ZX, Ren QY, Fang CS, Dou SX, Appl. Phys. Lett. 2015; 106:182405.

[37] Onodera H, Ohashi M, Yamauchi H, Yamaguchi Y, J. Magn. Magn. Mater. 1992;109:249.

[38] Ranke PJ von, Pecharsky VK, Gschneidner KA Jr. Phys. Rev. B 1998;58:12110.

[39] Singh NK, Kumar P, Suresh KG, Nigam AK. J. Appl. Phys. 2009;105:023901.

[40] Wang JL, Marquina C, Ibarra MR, Wu GH. Phys. Rev. B 2006;73:094436.

[41] Banerjee SK. Phys. Lett. 1964;12:16.

[42] Gerasimov EG, Kanomata T, Gaviko VS, Physica B 2007;390:118.

[43] Pathak AK, Paudyal D, Mudryk Y, Gschneidner, Jr K A, Pecharsky VK. Phys. Rev. Lett. 2013;110:186405.

[44] Singh NK, Agarwal S, Suresh KG. Phys. Rev. B 2005;72:014452.

[45] Gupta S, Suresh KG, Lukoyanov AV. J. Mater Sci. 2015;50:5723.

[46] Li L, Nishimura K. J. Appl. Phys. 2009;106:023903.

[47] Tishin AM, Spichkin YI. CRC Press 2003.

[48] Toulemonde O, Roussel P, Isnard O, Andre’ G, Mentre O. Chem. Mater. 2010;22:3807.

[49] Singh NK, Suresh KG, Nirmala R, Nigam AK, Malik SK. J. Magn. Magn. Mater. 2006;302:302.

\section{Figure Captions:}

Fig. 1. (left) A schematic of the crystal structure of $\mathrm{HoMn}_{2} \mathrm{Si}_{2}$. The Ho atoms are coloured violet, the $\mathrm{Mn}$ are green and $\mathrm{Si}$ are silver. (right) The Rietveld refinement of the $\mathrm{x}$ ray powder diffraction data of $\mathrm{HoMn}_{2} \mathrm{Si}_{2}$ in $\mathrm{I} 4 / \mathrm{mmm}$ symmetry. 
Fig. 2. The magnetic response of $\mathrm{HoMn}_{2} \mathrm{Si}_{2}$ Shows the magnetization at 500 Oe where magnetic transitions at $80 \mathrm{~K}, 15 \mathrm{~K}$ and $3.5 \mathrm{~K}$ are identified. The top inset shows the thermal hysteresis is visible in magnetization below $20 \mathrm{~K}$. The bottom inset shows the magnetic hysteresis loop of the compound at $2 \mathrm{~K}$ in low magnetic field.

Fig. 3. (a) Magnetic field dependence of the magnetization for $\mathrm{HoMn}_{2} \mathrm{Si}_{2}$ in the temperature range of 2-40 K with applied field of $5 \mathrm{~T}$. (b) The corresponding Arrott plots of $\mathrm{M}^{2}$ versus H/M.

Fig. 4. Temperature dependence of heat capacity for $\mathrm{HoMn}_{2} \mathrm{Si}_{2}$ for the temperature range from 0.5 to $150 \mathrm{~K}$. (a) Enlarge low temperature part to show more visible transitions. (b) Low temperature $\left(0.5\right.$ to $3 \mathrm{~K}$ ) heat capacity graph. (c) Shows $\mathrm{C}_{\mathrm{p}} / \mathrm{T}$ versus $\mathrm{T}^{2}$ at temperatures below $12 \mathrm{~K}$.

Fig. 5. Magnetic entropy changes of $\mathrm{HoMn}_{2} \mathrm{Si}_{2}$ calculated from magnetizations as a function of temperature for different magnetic field changes up to $5 \mathrm{~T}$.
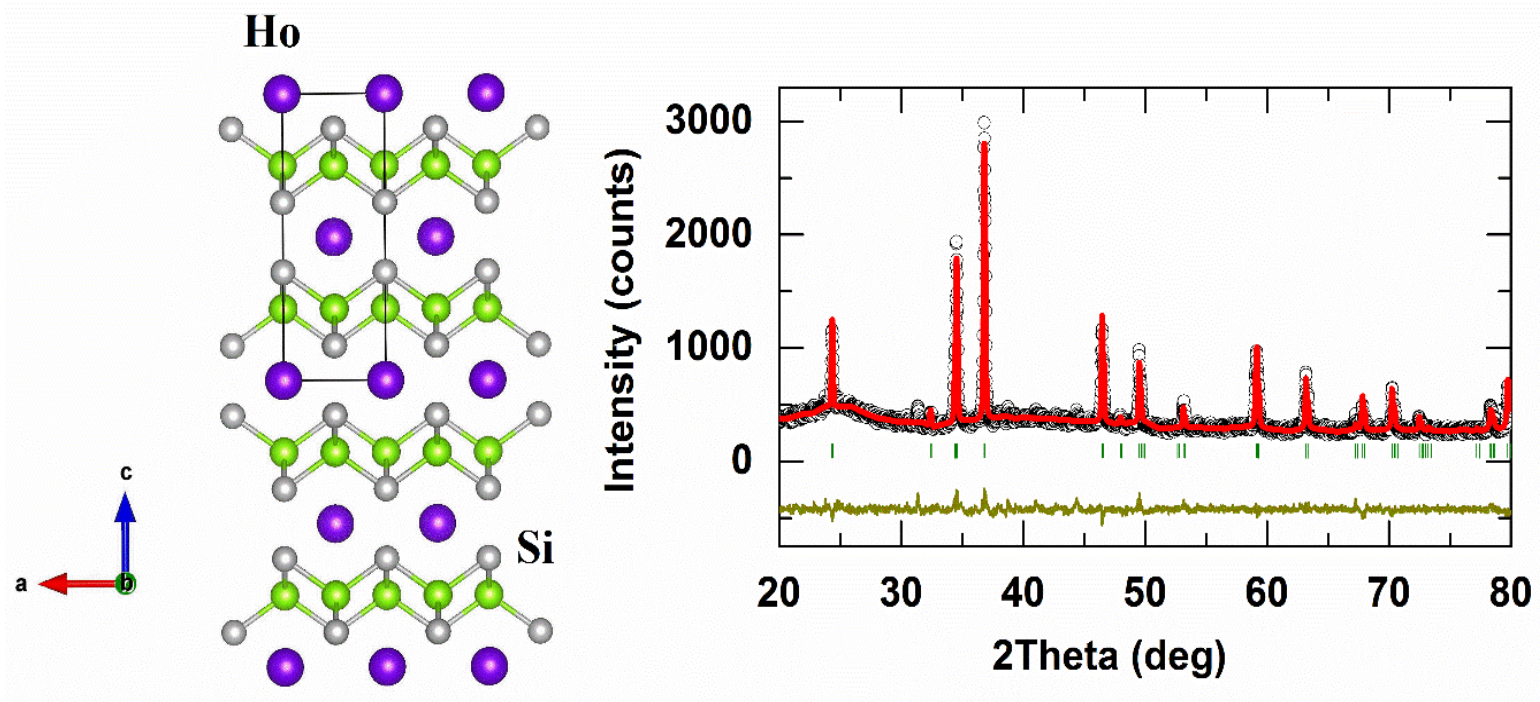

Fig. 1. Debnath et al. 


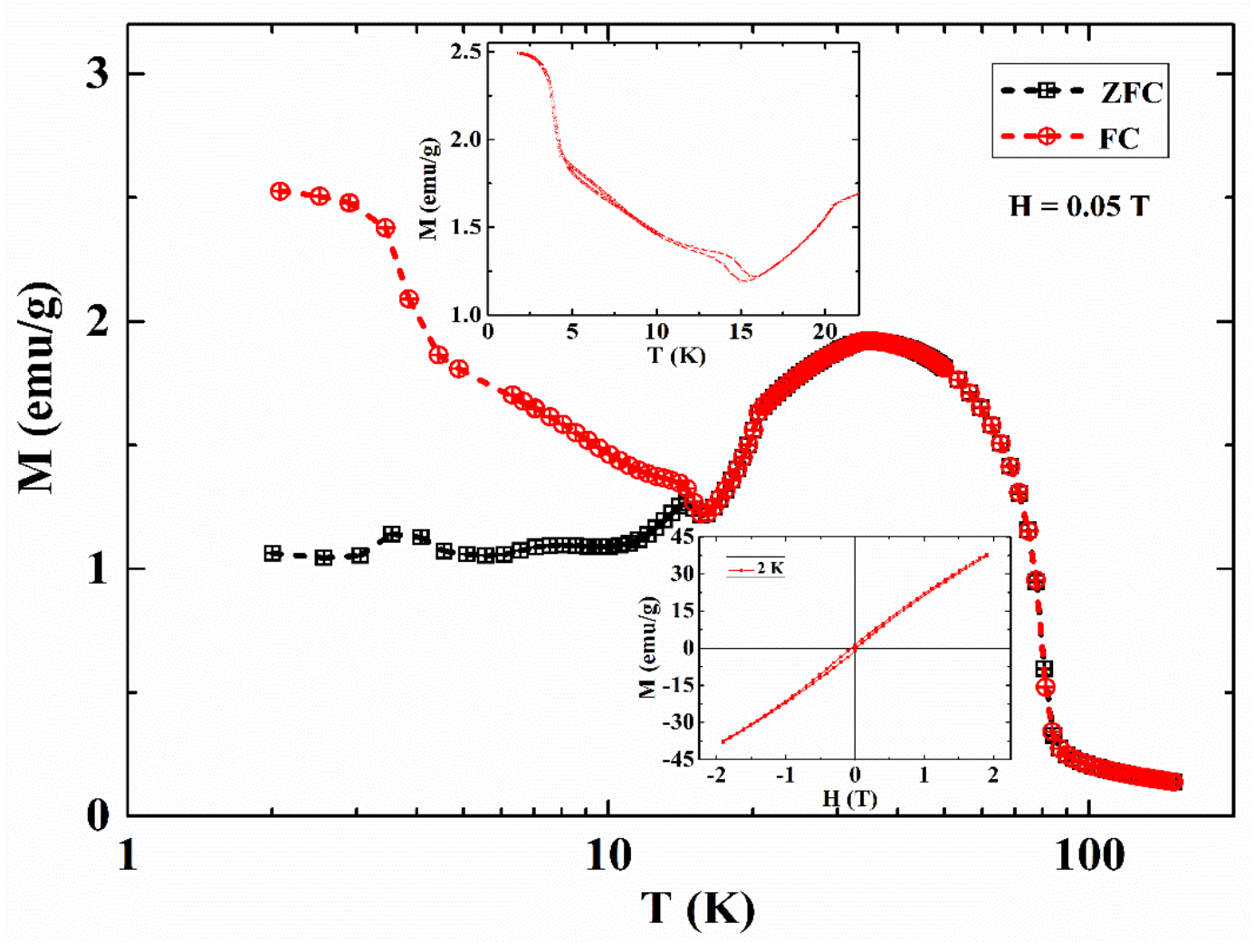

Fig. 2. Debnath et al.
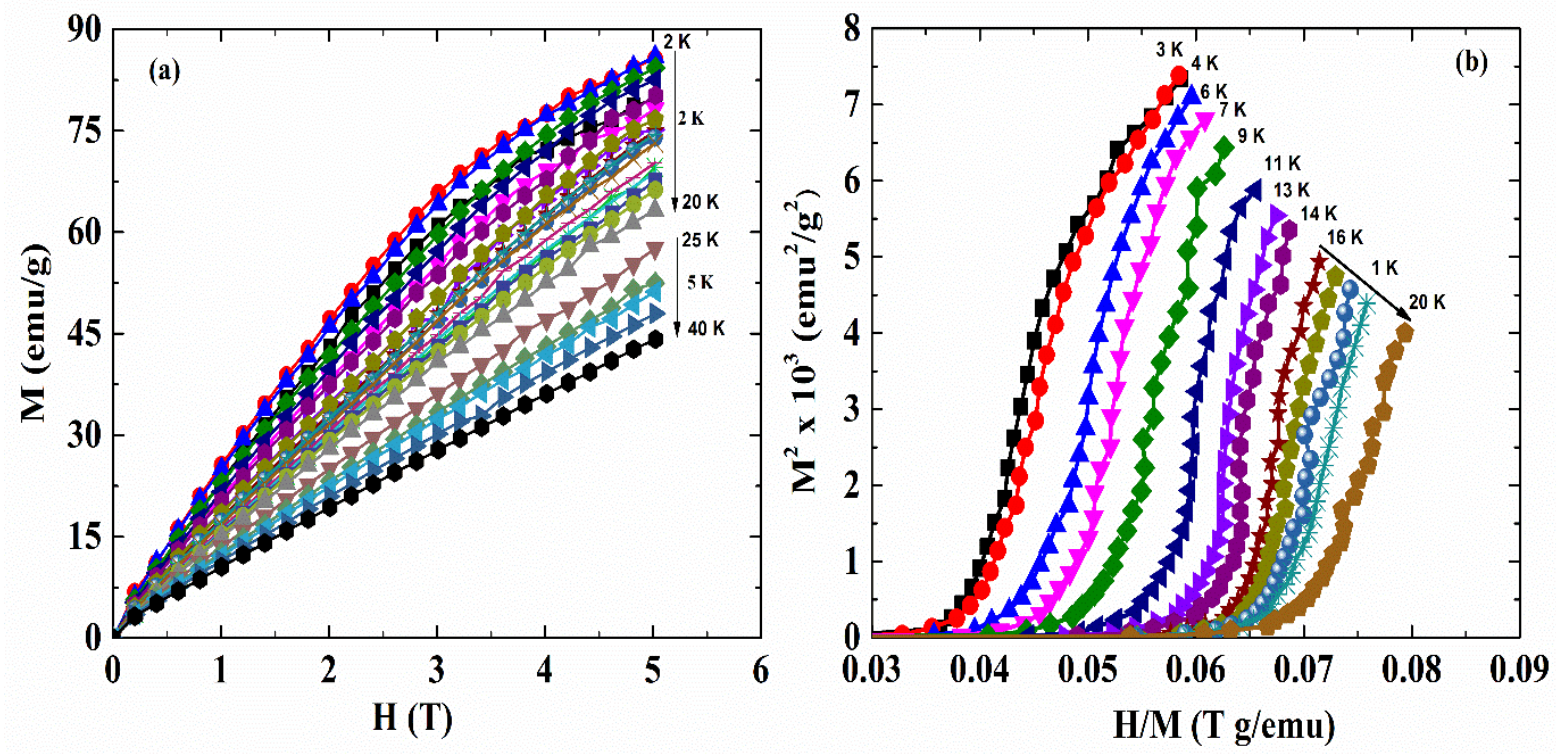

Fig. 3. Debnath et al. 


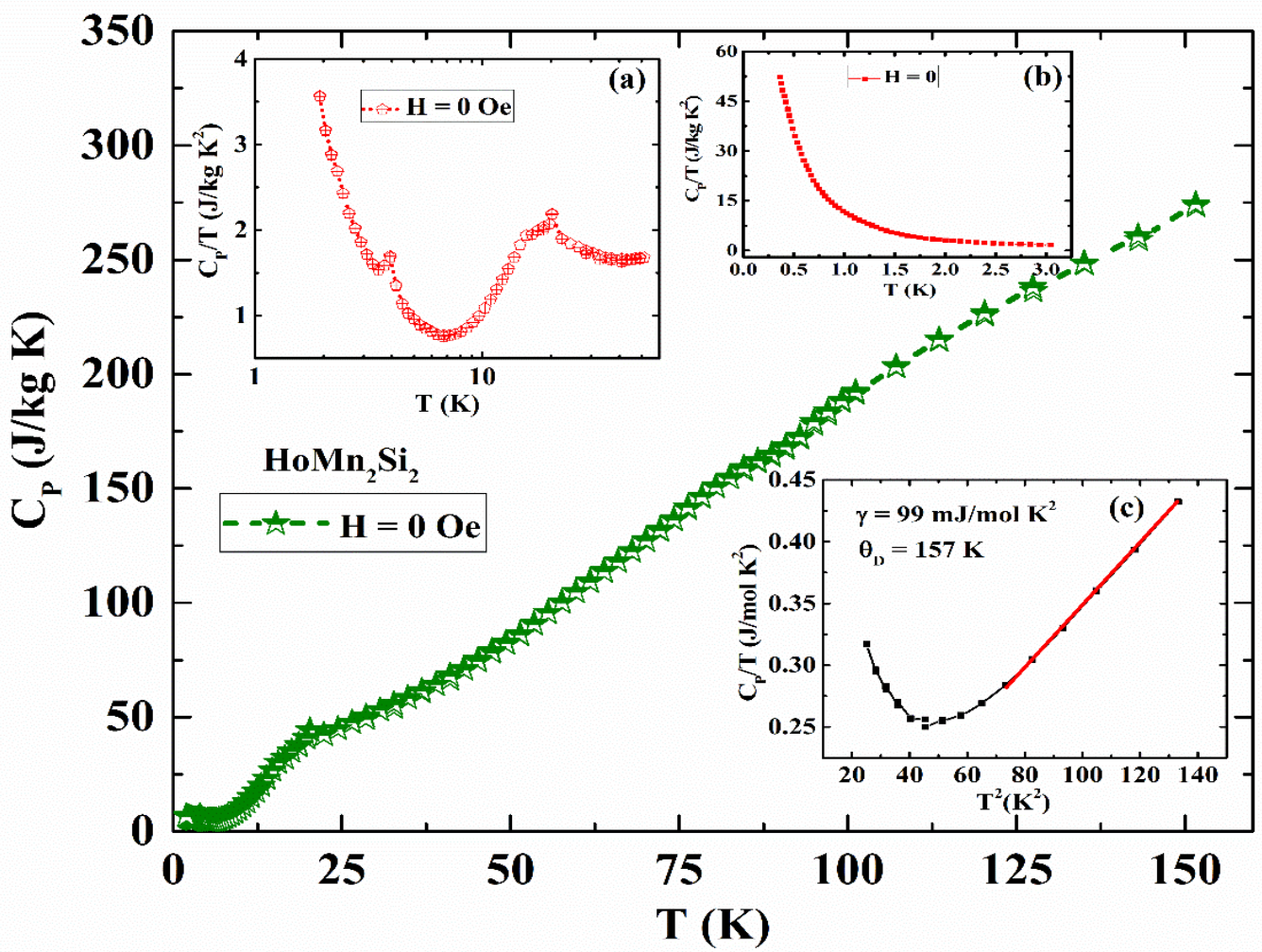

Figure 4 Debnath et al.

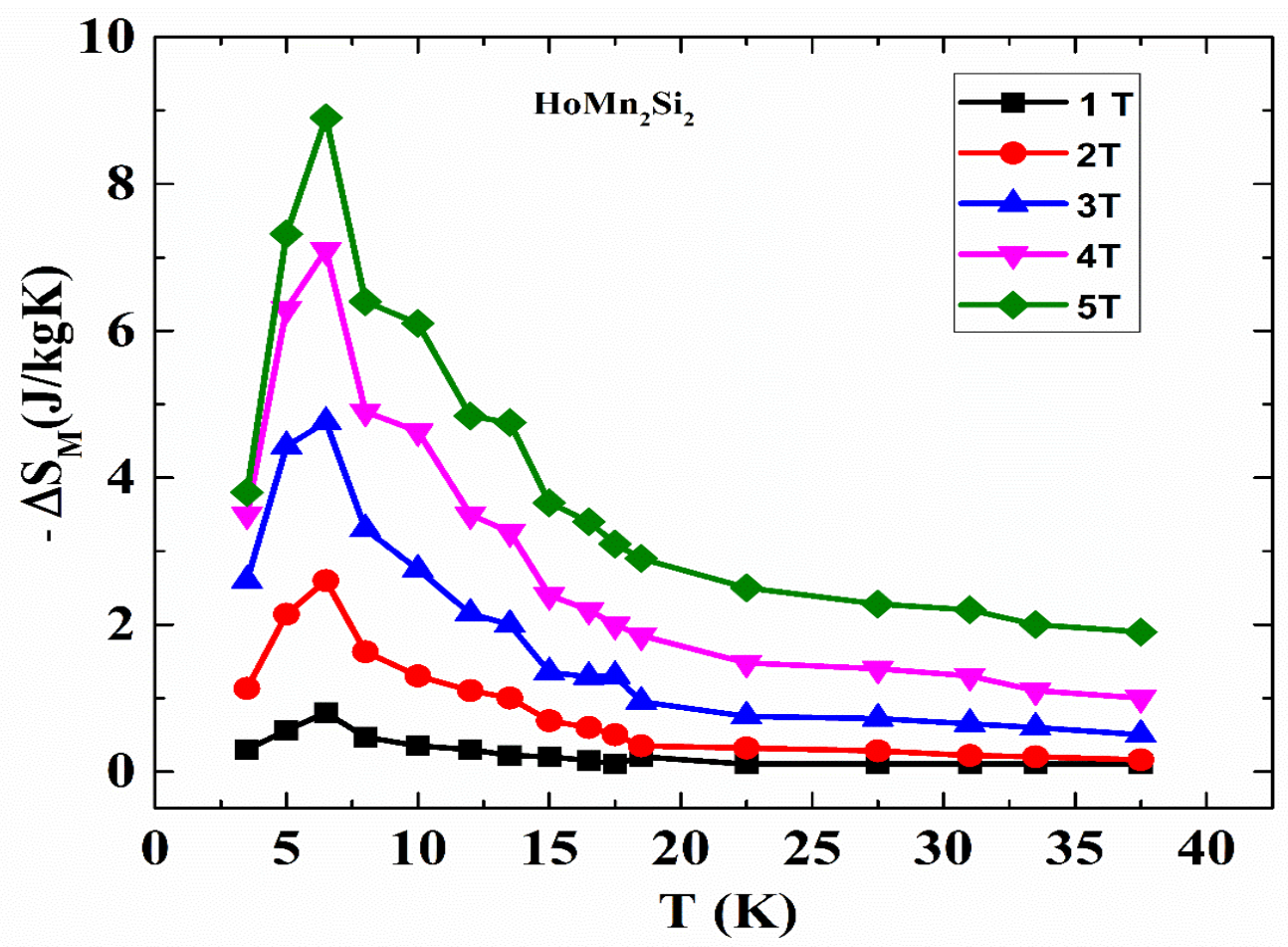

Fig. 5. Debnath et al. 